\title{
Extremalprobleme für eine Familie schlichter Funktionen
}

\author{
Renate MCLAughlin
}

\section{Einleitung}

Es sei $B$ ein Punkt im Kreisring $R=\left\{z: 0<r_{0}<|z|<1\right\}$, und $b$ sei ein Punkt in der offenen Kreisscheibe $D=\{w:|w|<1\}$. Wir betrachten die Klasse $F_{B, b}$ von in $R$ schlichten holomorphen Funktionen $f$, die den Bedingungen

(i) $|f(z)|<1$ für $z \in R, \lim _{|z| \rightarrow 1}|f(z)|=1$,

(ii) $f(B)=b$

genügen. Offensichtlich kann jede Funktion $f \in F_{B, b}$ analytisch in den Kreisring $\left\{z: r_{0}<|z|<1 / r_{0}\right\}$ fortgesetzt werden, und es gelten die Beziehungen

$$
|f(z)|=1 \text { für }|z|=1 \text { und } \overline{f\left(\frac{1}{\bar{z}}\right)}=\frac{1}{f(z)} .
$$

Wir bezeichnen das Kontinuum $D-f(R)$ mit $\Gamma_{f}$.

Man kann leicht zeigen, daß die Menge $F_{B, b}$ kompakt ist. Folglich existieren Extremalfunktionen, und wir können Extremalprobleme in $F_{B, b}$ mit Variationsmethoden lösen. Wir werden für die Klasse $F_{B, b}$ eine Variationsmethode entwickeln, die auf einer Variationsmethode von Duren und Schiffer [2] beruht, und wir werden sie auf die folgenden Extremalprobleme in $F_{B, b}$ anwenden.

1. Wir fragen nach der Verzerrung im Punkte $B$; d.h., wir fragen nach den Extremwerten der Größe $\left|f^{\prime}(B)\right|\left(f \in F_{B, b}\right)$.

2. Wir fragen nach der Drehung im Punkt $B$; d.h., wir fragen nach dem Maximum des Argumentes von Ableitungen in $B$.

3. Wir fragen nach den Extremwerten der Größe

$$
\mathfrak{R}\left(B \frac{f^{\prime}(B)}{f(B)-c}\right) \quad\left(|c|<1, c \neq b, f \in F_{B, b}\right) .
$$

Dieses Problem ist verwandt mit der Frage nach einer "Sternschranke".

4. Wir fragen nach den Extremwerten der Größe

$$
\mathfrak{R}\left(1+B \frac{f^{\prime \prime}(B)}{f^{\prime}(B)}\right) \quad\left(f \in F_{B, b}\right) .
$$

Dieses Problem ist verwandt mit der Frage nach einer "Rundungsschranke“. 
5. Wir fragen, welche Werte die Krümmung der Bilder von Kreisen $|z|=r\left(r_{0}<r<1\right)$ in einem festen Punkt annehmen kann.

Für jedes Extremalproblem erhalten wir eine Differentialgleichung, die von dem Kontinuum $\Gamma_{g}$ ( $g$ ist eine Extremalfunktion) erfüllt sein muß. Für den Spezialfall $b=0$ kann man leicht explizite Lösungen der Differentialgleichung angeben. Insbesondere erhalten wir in Frage 1 für $b=0$ die klassischen Resultate, nämlich eine Funktion $g$, für die $\Gamma_{g}$ ein Kreisbogen ist, und eine Funktion $h$, für die $\Gamma_{h}$ eine radiale Strecke ist.

Im Gegensatz zu anderen Klassen von schlichten holomorphen Funktionen in $R$, die in der Literatur behandelt sind (z. B. [1 bis 6, 8, 11]), gibt es in $F_{B, b}$ keine Normalisierung für das Kontinuum $\Gamma_{f}$. Dieser Nachteil wird aber dadurch aufgehoben, daß viele Fragen, die in den anderen Klassen recht komplizierte Antworten haben, in $F_{B, b}$ leicht zugänglich sind.

Einige Resultate dieser Arbeit sind in der Dissertation [11] enthalten, die unter der Leitung von Prof. Dr. P. L. Duren geschrieben wurde.

\section{Eine Variationsmethode für $\boldsymbol{F}_{\boldsymbol{B}, \boldsymbol{b}}$}

Für jede Funktion $f \in F_{B, b}$ werden wir eine Schar von Funktionen $U_{\rho}(w)$ $\left(0<\rho<\rho_{0}\right)$ erhalten, die den folgenden Bedingungen genügen:

(i) $U_{\rho} \circ f \in F_{B, b}$,

(ii) $\lim _{\rho \rightarrow 0} U_{\rho}(w)=w$

(iii) $\left|U_{\rho}(w)\right|<1$ für $w \in f(R),\left|U_{\rho}(w)\right|=1$ auf $|w|=1$,

(iv) $U_{\rho}(b)=b$.

Es sei nun $f$ eine Funktion in $F_{B, b}$. Wir wählen einen Punkt $w_{0}$ in $\Gamma_{f}$. Die Funktion

$$
\phi(w)=\frac{w-b}{1-\bar{b} w}
$$

ist schlicht in $D \cup C(C=\{w:|w|=1\})$, bildet $D$ auf $D$ und $C$ auf $C$ ab und genügt der Bedingung $\phi(b)=0$. Wie Duren und Schiffer [2] gezeigt haben, gibt es für jedes $w_{0} \in \Gamma_{f}$ und jede genügend kleine, positive Zahl $\rho$ Funktionen $G$ und $\chi$ mit den folgenden Eigenschaften: $G$ ist eine schlichte holomorphe Funktion in der Menge

$$
D-\left\{w \in \phi\left(\Gamma_{f}\right):\left|w-\phi\left(w_{0}\right)\right| \leqq \rho\right\},
$$

$G(0)=0$, und $G$ erhält den Einheitskreis bis zur Ordnung $\rho^{3}$. Das Bild von $C$ unter $G$ sei $C_{\rho}$.

Die Funktion $\chi$ ist schlicht und holomorph im Innern von $C_{\rho}$ und bildet das Innere von $C_{\rho}$ so auf $D \mathrm{ab}$, daß $\chi(0)=0$. Man betrachte nun die Funktion

$$
U_{\rho}(w)=\phi^{-1} \circ \chi \circ G \circ \phi(w) .
$$

Offensichtlich ist $U_{\rho}$ schlicht und holomorph im Bildbereich von $f$, und $U_{\rho}$ genügt Bedingungen (i) bis (iv).

23 Math. Z., Bd. 118 
Eine Rechnung zeigt, da $\beta$

$$
\begin{aligned}
U_{\rho}(w)=w[1 & +\frac{a \rho^{2}\left(1-\bar{b} w_{0}\right)^{2}(w-b)(1-\bar{b} w)^{2}}{\left(1-|b|^{2}\right)^{2}\left(w_{0}-b\right) w\left(w-w_{0}\right)} \\
& \left.-\frac{\bar{a} \rho^{2}\left(1-b \bar{w}_{0}\right)^{2}(w-b)^{2}(1-\bar{b} w)}{\left(1-|b|^{2}\right)^{2}\left(\bar{w}_{0}-\bar{b}\right) w\left(1-\bar{w}_{0} w\right)}\right]+O\left(\rho^{3}\right)
\end{aligned}
$$

( $a$ ist eine komplexe Konstante $(|a|<1)$, die von $\rho$ abhängt).

Wir bemerken, daß die Ableitung von $U_{\rho}(w)$ in $b$ besonders einfach ist:

$$
U_{\rho}^{\prime}(b)=1-a \rho^{2}\left(\frac{1-\bar{b} w_{0}}{b-w_{0}}\right)^{2}+O\left(\rho^{3}\right) .
$$

Für den Fall $b=0$ stehen uns zwei Klassen von Funktionen zur Verfügung. Die Klasse $F_{B, 0}$ enthält mit jeder Funktion $f$ auch alle Drehungen $e^{i \theta} f$. Wenn wir Drehungen ausschließen möchten, benutzten wir die Unterklasse

$$
F_{B, 0}^{*}=\left\{f: f \in F_{B, 0} \text { und } f(1)=1\right\} \text {. }
$$

Als Variationsfunktionen in $F_{B, 0}^{*}$ erhält man

$$
U_{\rho}^{*}(w)=\frac{1}{U_{\rho}(1)} U_{\rho}(w)
$$

wobei in $U_{\rho}(1)$ und $U_{\rho}(w)$ für $b$ der Wert 0 eingesetzt ist.

\section{Verzerrung im Punkt $B$}

Nehari [12, Kapitel VII] betrachtete Familien von Funktionen, die einen mehrfach zusammenhängenden Bereich $A$ so abbilden, daß ein fester Punkt $u \in A$ in den Punkt $w=0$ übergeht. Er fand, daß das Maximum der Größe $\left|f^{\prime}(u)\right|$ von einer Kreisbogenschlitzabbildung angenommen wird, und daß eine Radialschlitzabbildung das Minimum der Größe $\left|f^{\prime}(u)\right|$ liefert. Für den Fall $b=0$ erhalten wir diese Resultate mit unserer Methode. Unsere Methode ist weniger anschaulich als die von Nehari, aber sie hat den Vorteil, daß man sie auf viele verschiedene Probleme anwenden kann.

Zunächst untersuchen wir die Größe $\max _{f \in F_{B, b}}\left|f^{\prime}(B)\right|$. Da die Familie $F_{B, b}$ kompakt ist, existiert eine Funktion $g \in F_{B, b}$ mit der Eigenschaft, daß

$$
\left|g^{\prime}(B)\right|=\max _{f \in F_{B, b}}\left|f^{\prime}(B)\right| .
$$

Folglich gilt die Ungleichung $\left|g^{\prime}(B)\right| \geqq\left|\left(U_{\rho} \circ g\right)^{\prime}(B)\right|=\left|U_{\rho}^{\prime}(b) g^{\prime}(b)\right|$. Eine kurze Rechnung führt von dieser Ungleichung zu der Bedingung

$$
\mathfrak{R}\left[-a \rho^{2}\left(\frac{1-\bar{b} w_{0}}{b-w_{0}}\right)^{2}+O\left(\rho^{3}\right)\right] \leqq 0 .
$$

Wir benutzen nun ein Lemma von Schiffer $([7,13])$. Es ist das wichtigste Hilfsmittel dieser Variationsmethode. Das Lemma von Schiffer besagt Folgendes. 
Es sei $g$ eine Extremalfunktion. Falls jede Variationsfunktion $U_{\rho}$ zu einer Ungleichung der Form

$$
\Re\left\{a \rho^{2} s\left(w_{0}\right)+O\left(\rho^{3}\right)\right\} \leqq 0
$$

führt $\left(s(w)\right.$ ist eine holomorphe Funktion auf $\Gamma_{g}$, die nicht identisch verschwindet), so besteht $\Gamma_{\mathrm{g}}$ aus analytischen Kurven $w(t)$, die der Differentialgleichung

genügen.

$$
w^{\prime}(t)^{2} s(w(t))>0
$$

Von Ungleichung (2) erhalten wir also die Differentialgleichung

$$
w^{\prime}(t)^{2}\left(\frac{1-\bar{b} w(t)}{b-w(t)}\right)^{2}<0
$$

für das Kontinuum $\Gamma_{g}$, wobei $g$ eine Extremalfunktion für das Maximumproblem ist.

Für den Spezialfall $b=0$ hat die Differentialgleichung (3) die besonders einfache Form $\left(w^{\prime}(t) / w(t)\right)^{2}<0$, und die allgemeine Lösung ist

$$
w(t)=K e^{i t} \quad(K \text { eine komplexe Konstante }) .
$$

Also ist $\Gamma_{g}$ ein Bogen eines Kreises um den Nullpunkt.

Für allgemeines $b$ müssen die Lösungskurven von (3) der Funktionalgleichung

$$
e^{-\bar{b} w}(w-b)^{1-|b|^{2}}=K e^{i t}
$$

genügen. Die Kurven sind aber nicht mehr einfach zu beschreiben.

Wenn wir das Minimalproblem $\min _{f \in F_{B, b}}\left|f^{\prime}(B)\right|$ betrachten, erhalten wir die Differentialgleichung

$$
w^{\prime}(t)^{2}\left(\frac{1-\bar{b} w(t)}{b-w(t)}\right)^{2}>0 .
$$

Für allgemeines $b$ sind die Lösungskurven kompliziert, aber für $b=0$ erhalten wir die Kurvenschar

$$
w(t)=K e^{t} \quad(K \text { komplex }) .
$$

Für den Spezialfall $b=0$ können wir die Extremalfunktionen selbst angeben und beide Extremalprobleme quantitativ lösen. Es sei

$$
A_{1}=\min _{f \in F_{B}, 0}\left|f^{\prime}(B)\right| \text { und } A_{2}=\max _{f \in F_{B, 0}}\left|f^{\prime}(B)\right| .
$$

Wir erhalten $A_{1}$ von einer Funktion, die $R$ auf die Einheitskreisscheibe abbildet, von der eine radiale Strecke entfernt ist. Eine solche Abbildung wurde von Gehring und af Hällström [5] konstruiert. Ohne Beschränkung der Allgemeinheit dürfen wir annehmen, daß die radiale Strecke auf der negativen reellen Achse liegt und da $B$ B positiv ist. Wir benötigen die folgenden $23^{*}$ 
Konstanten:

$$
t=2 r_{0} \prod_{n=1}^{\infty}\left(\frac{1+r_{0}^{8 n}}{1+r_{0}^{8 n-4}}\right)^{2}, \quad k=\frac{1-t}{1+t}, \quad \text { und } \quad d=\frac{K}{-\log r_{0}}
$$

wobei $K=K(k)=\int_{0}^{\pi / 2}\left(1-k^{2} \sin ^{2} t\right)^{-\frac{1}{2}} d t$. Die Konstanten $t, k$ und $d$ sind von dem Kreisring $R$ völlig bestimmt. Wir betrachten nun die Komposition der folgenden Abbildungen:

$$
\zeta(z)=\log z, \quad \omega(\zeta)=\operatorname{sn}(d \zeta, k), \quad w(\omega)=\frac{-\operatorname{sn}(d \log B, k)+\omega}{-\operatorname{sn}(d \log B, k)-\omega}
$$

( $\log z$ ist der Hauptzweig der Logarithmusfunktion). Die Funktion $\zeta(z)$ bildet die obere Hälfte des Kreisringes $R$ auf ein Rechteck im zweiten Quadranten $\mathrm{ab}$, die Funktion $\omega(\zeta)$ bildet dieses Rechteck auf den ganzen zweiten Quadranten der komplexen Ebene $a b$, und $w(\omega)$ bildet den zweiten Quadranten auf die obere Hälfte des Einheitskreises ab. Wie die untere Hälfte von $R$ abgebildet wird, folgt von der Symmetrie der einzelnen Funktionen.

Die Funktion

$$
g(z)=\frac{\operatorname{sn}(d \log B, k)-\operatorname{sn}(d \log z, k)}{\operatorname{sn}(d \log B, k)+\operatorname{sn}(d \log z, k)}
$$

gehört offensichtlich zur Klasse $F_{B, 0}$, und sie ist eine Extremalfunktion. Also ist

Eine Rechnung zeigt, daß

$$
A_{1}=\left|g^{\prime}(B)\right| \text {. }
$$

$$
g^{\prime}(B)=-\frac{d}{2 B} \frac{\operatorname{cn}(d \log B, k) \mathrm{dn}(d \log B, k)}{\operatorname{sn}(d \log B, k)}>0 .
$$

Die Lösung des Maximumproblems ist nicht viel schwieriger. Wir dürfen wieder annehmen, daß $B$ positiv ist.

Es sei $\tau$ ein Punkt auf der positiven imaginären Achse, und $T$ sei das Rechteck mit Ecken $0,1,1+\tau / 2$ und $\tau / 2$. In [10, S. 362] wurde gezeigt, daß die Funktion

$$
\omega(\zeta)=C \frac{\vartheta_{1}(\zeta+i \sigma)}{\vartheta_{1}(\zeta-i \sigma)}
$$

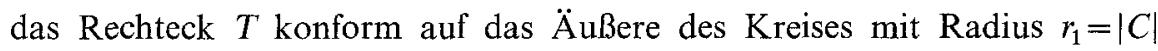
abbildet, von dem ein Bogen des konzentrischen Kreises mit Radius $r_{2}=|C| e^{2 \pi \sigma}$ entfernt ist. Hierbei ist $\vartheta_{1}(\zeta)$ die Jacobische Thetafunktion mit Parameter $\tau$, und $i \sigma(0<\sigma<|\tau| / 2)$ ist eine Zahl mit der Eigenschaft, daß $\omega(i \sigma)=\infty$. Wir betrachten nun die Komposition der folgenden Abbildungen:

$$
\zeta(z)=\frac{1}{2 \pi i} \log z
$$


( $\log z$ ist der Hauptzweig der Logarithmusfunktion),

$$
\omega(\zeta)=e^{i \theta} \frac{\vartheta_{1}(\zeta+i|\log B| / 2 \pi)}{\vartheta_{1}(\zeta-i|\log B| / 2 \pi)}
$$

(der Parameter $\tau$ der Thetafunktion ist $\tau=i\left|\log r_{0}\right| / \pi$, und $\theta$ ist eine reelle Konstante), und

$$
w(\omega)=\frac{1}{\omega} .
$$

Die Funktion $\zeta(z)$ bildet den Kreisring $R$ auf das Rechteck $T$ ab, wobei $\tau=i\left|\log r_{0}\right| / \pi$. Die Funktion $\omega(\zeta)$ bildet $T$ auf das Äußere des Einheitskreises $a b$, von dem ein Bogen des Kreises mit Radius 1/B entfernt ist. Die Komposition dieser Abbildungen

$$
g(z)=e^{-i \theta} \frac{\vartheta_{1}\left(\frac{1}{2 \pi i} \log z-\frac{i}{2 \pi}|\log B|\right)}{\vartheta_{1}\left(\frac{1}{2 \pi i} \log z+\frac{i}{2 \pi}|\log B|\right)}
$$

gehört zur Klasse $F_{B, 0}$ und ist eine Extremalfunktion für jedes reelle $\theta$. Wir bemerken, da $\beta$ der Kreisbogen $\Gamma_{\mathrm{g}}$ auf dem Kreis $|z|=B$ liegt.

Eine Rechnung zeigt, daß die Zahl $A_{2}$ durch den folgenden Ausdruck gegeben ist:

$$
A_{2}=\left|g^{\prime}(B)\right|=\frac{r_{0}^{\frac{1}{2}}}{\pi B}\left(\prod_{n=1}^{\infty}\left(1-r_{0}^{2 n}\right)\right)^{3}\left|\vartheta_{1}\left(\frac{1}{\pi i} \log B\right)\right|^{-1}
$$

\section{Die Drehung im Punkt $B$}

Wir setzen $b=0$ und betrachten nur normalisierte Funktionen, für die $f(1)=1$ gilt. Für jede solche Funktion $f \in F_{B, 0}^{*}$ haben die Tangentenvektoren an den Kreis $|z|=1$ im Punkt $z=1$ und an den Kreis $|w|=1$ in $w=1$ das gleiche Argument. Für jede Funktion $f \in F_{B, 0}^{*}$ können wir also den Zweig der Logarithmusfunktion wählen, für $\operatorname{den} \mathfrak{I} \log f^{\prime}(1)=0$ gilt. Für diesen Zweig fragen wir nach den Größen

$$
\max _{f \in F_{B, 0}^{*}} \mathfrak{I} \log f^{\prime}(B) \text { und } \min _{f \in F_{B, 0}^{*}} \mathfrak{I} \log f^{\prime}(B) .
$$

Die Reihenentwicklung einer Funktion in $F_{B, 0}^{*}$ um $B$ zeigt, daß wenn $g(z)$ eine Extremalfunktion für das Maximumproblem ist, so ist $\overline{g(\bar{z})}$ eine Extremalfunktion für das Minimumproblem. Es genuigt also, das Maximumproblem zu betrachten.

Es sei $g$ eine Extremalfunktion für das Maximumproblem. Ein Vergleich von $g^{\prime}(B)$ mit der Ableitung in $B$ der Funktion $\frac{1}{U_{\rho}(1)} U_{\rho}(g(z))$ führt zu der
Ungleichung

$$
\mathfrak{I} \log \frac{U_{\rho}^{\prime}(0)}{U_{\rho}(1)} \leqq 0
$$


Nach einer kurzen Rechnung erhalten wir die Bedingung

$$
\mathfrak{R}\left[a \rho^{2} \frac{i\left(1+w_{0}\right)}{w_{0}^{2}\left(1-w_{0}\right)}+O\left(\rho^{3}\right)\right] \leqq 0 .
$$

Schiffers Lemma besagt nun, daß das Kontinuum $\Gamma_{g}$ eine analytische Kurve ist, die der Differentialgleichung

genügt.

$$
w^{\prime}(t)^{2} \frac{i(1+w(t))}{w(t)^{2}(1-w(t))}>0
$$

Die Lösungskurven von (5) sind symmetrisch in bezug auf den Einheitskreis $|w|=1$. In der Nähe des Nullpunkts sind sie lokal wie logarithmische Spiralen. Die obere Hälfte des Einheitskreises, $w(t)=e^{i t}(0<t<\pi)$, ist eine Lösungskurve, aber die untere Hälfte des Einheitskreises schneidet Lösungskurven senkrecht. Die Lösungskurven verlassen den Nullpunkt als Spiralen, winden sich um den Nullpunkt in der oberen Hälfte von $D$ und verlassen $D$ in der unteren Halbebene.

Benutzen wir die spezielle Parameterdarstellung $w(t)=g\left(r_{0} e^{i t}\right)$ für die Kurve $\Gamma_{g}$, so können wir von (5) die Differentialgleichung

$$
\begin{aligned}
g^{\prime}(z)^{2} & \frac{1+g(z)}{g(z)^{2}(1-g(z))} \\
= & i z^{-2}\left[K+2 i g^{\prime}(1) \zeta(\log z)-i A \zeta\left(\log \frac{z}{B}\right)-i \bar{A} \zeta(\log z \bar{B})\right. \\
& \left.\quad-i \wp\left(\log \frac{z}{B}\right)+i \wp(\log z \bar{B})\right]
\end{aligned}
$$

für die Extremalfunktion $g$ erhalten. Hier sind $\zeta(u)$ und $\wp(u)$ die Weierstraßschen $\zeta$ - und $\wp$-Funktionen mit Perioden $2 n \pi i+2 m \log r_{0} ; K$ ist eine reelle Konstante; $A=1+B g^{\prime \prime}(B) / g^{\prime}(B)+2 B g^{\prime}(B) ;$ und $g^{\prime}(1)=\Re A$. Man kann leicht Beziehungen zwischen den Parametern $K, A$ und $g^{\prime}(1)$ finden, aber anscheinend ist es schwierig, diese Parameter explizit zu bestimmen.

\section{Maximum und Minimum der Größe $\mathfrak{R}\left(B \frac{f^{\prime}(B)}{f(B)-c}\right)$}

Man wähle einen Punkt $c$ in $D$. Jede Funktion der Klasse $F_{B, b}$ bildet den Einheitskreis $|z|=1$ auf den Einheitskreis $|w|=1 \mathrm{ab}$. Das Innere des Bildkreises $|w|=1$ ist sternförmig in bezug auf $c$. Deshalb gehen Kreise $|z|=r$ in Kurven über, deren Inneres ebenfalls sternförmig in bezug auf $c$ ist, wenn nur $r$ genügend groß ist $(r<1)$. Eine Funktion $f$ bildet den Kreis $|z|=r$ auf eine Kurve ab, deren Inneres sternförmig in bezug auf $c$ ist, falls das Argument von $f\left(r e^{i t}\right)-c$ monoton mit $t$ wächst, das heißt, falls

$$
\Re\left(z \frac{f^{\prime}(z)}{f(z)-c}\right) \geqq 0 \quad(|z|=r) .
$$


In diesem Abschnitt fragen wir nach dem Maximum und Minimum der Größe $\mathfrak{R}\left(B f^{\prime}(B) /(f(B)-c)\right)$ für Funktionen der Klasse $F_{B, b}(b \neq c)$.

Wegen der Kompaktheit der Klasse $F_{B, b}$ existiert eine Funktion $g \in F_{B, b}$ $(b \neq c)$ mit der Eigenschaft, daß

$$
\mathfrak{R}\left(B \frac{g^{\prime}(B)}{g(B)-c}\right)=\max _{f \in F_{B, b}} \mathfrak{R}\left(B \frac{f^{\prime}(B)}{f(B)-c}\right) .
$$

Ein Vergleich von $g$ mit den Funktionen $U_{\rho} \circ g$ führt zu der Ungleichung

$$
\mathfrak{R}\left[a \rho^{2} \frac{B}{b-c} g^{\prime}(B)\left(\frac{1-\bar{b} w_{0}}{b-w_{0}}\right)^{2}+O\left(\rho^{3}\right)\right] \geqq 0 .
$$

Deshalb ist das Kontinuum $\Gamma_{g}$ eine analytische Kurve, die der Differentialgleichung

genügt.

$$
w^{\prime}(t)^{2} \frac{B}{b-c} g^{\prime}(B)\left(\frac{1-\bar{b} w(t)}{b-w(t)}\right)^{2}<0
$$

Von der Theorie der quadratischen Differentiale [9, S. 32] folgt, daß das Verhalten der Lösungskurven zu (6) in der Nähe von $b$ von dem Parameter $\ell=i\left[(b-c) / B g^{\prime}(B)\right]^{\frac{1}{2}}$ abhängt. Im Fall $\mathfrak{R} \ell \neq 0$ und $\mathfrak{\Im} \ell \neq 0$ verhalten sich die Lösungskurven zu (6) in der Nähe von $b$ wie logarithmische Spiralen. Im Fall $\mathfrak{R} \ell \neq 0, \mathfrak{J} \ell=0$ sind die Lösungskurven (lokal) Strahlen, die von $b$ ausgehen. Im Fall $\mathfrak{R} \ell=0, \mathfrak{J} \ell \neq 0$ sind die Lösungskurven (lokal) einfach geschlossene Kurven um $b$.

Wählen wir nun $B>0$ und $c<b=0$, und nehmen wir zusätzlich an, daß $g^{\prime}(B)>0$ gilt, so erhalten wir von (6) die Differentialgleichung

$$
\left(\frac{w^{\prime}(t)}{w(t)}\right)^{2}<0
$$

deren Lösungskurven Kreise um den Nullpunkt sind.

Für das Minimalproblem

$$
\min _{f \in F_{B, b}} \Re\left(B \frac{f^{\prime}(B)}{f(B)-c}\right)
$$

brauchen wir nur alle Ungleichheitszeichen umzukehren. Für den Spezialfall $B>0, c<b=0$ und $g^{\prime}(B)>0$ erhalten wir die Differentialgleichung

$$
\left(\frac{w^{\prime}(t)}{w(t)}\right)^{2}>0
$$

deren Lösungskurven Strahlen vom Nullpunkt sind.

Für die Normalisierung $B>0, c<b=0$ und $g^{\prime}(B)>0$ erreicht die Größe $\mathfrak{R}\left[B f^{\prime}(B) /(f(B)-c)\right]$ also ein Minimum für eine Funktion $g$, für die $\Gamma_{g}$ eine Strecke der negativen reellen Achse ist. Das Maximum wird erreicht für eine Funktion $h$, für die $\Gamma_{h}$ ein Kreisbogen (symmetrisch in bezug auf die reelle Achse) in der linken Halbebene ist. 


\section{Maximum und Minimum der Größe $\mathfrak{R}\left(1+B \frac{f^{\prime \prime}(B)}{f^{\prime}(B)}\right)$}

Jede Funktion der Klasse $F_{B, b}$ bildet den Einheitskreis $|z|=1$ auf den Einheitskreis $|w|=1 \mathrm{ab}$, dessen Inneres konvex ist. Für genügend große Werte von $r$ $(r<1)$ werden deshalb Kreise $|z|=r$ auf Kurven abgebildet, deren Inneres ebenfalls konvex ist. Das Bild eines Kreises $|z|=r$ unter einer Funktion $f$ ist konvex, falls $\Re\left[1+z f^{\prime \prime}(z) / f^{\prime}(z)\right] \geqq 0(|z|=r)$.

In diesem Abschnitt fragen wir, für welche Funktionen in $F_{B, b}$ die Größe $\mathfrak{R}\left[1+B f^{\prime \prime}(B) / f^{\prime}(B)\right]$ ein Maximum und Minimum erreicht.

Zunächst sei $g$ eine Funktion in $F_{B, b}$ mit der Eigenschaft

$$
\mathfrak{R}\left(1+B \frac{g^{\prime \prime}(B)}{g^{\prime}(B)}\right)=\max _{f \in F_{B, b}} \mathfrak{R}\left(1+B \frac{f^{\prime \prime}(B)}{f^{\prime}(B)}\right) .
$$

Ein Vergleich von $g$ mit der Funktion $U_{\rho} \circ g$ führt zu der Ungleichung

wo

$$
\mathfrak{R}\left[a \rho^{2} s\left(w_{0}\right)+O\left(\rho^{3}\right)\right] \leqq 0,
$$

$$
s(w)=\frac{1-\bar{b} w}{b-w}\left\{\frac{B g^{\prime}(B)}{(b-w)^{2}}(1-\bar{b} w)\left(1+|b|^{2}-2 \bar{b} w\right)+\overline{B g^{\prime}(B)}\right\} .
$$

Also ist das Kontinuum $\Gamma_{g}$ eine analytische Kurve, die der Differentialgleichung $w^{\prime}(t)^{2} s(w(t))>0$ genügt.

Für den Spezialfall $b=0$ wird die Differentialgleichung für $\Gamma_{g}$ besonders einfach, nämlich

$$
w^{\prime}(t)^{2} w(t)^{-3}\left[B g^{\prime}(B)+w(t)^{2} \overline{B g^{\prime}(B)}\right]<0 .
$$

Da die Klasse $F_{B, 0}$ mit jeder Funktion $f$ auch alle Funktionen $e^{i \theta} f(\theta$ reell) enthält, und da die Größe $\Re\left[1+B e^{i \theta} f^{\prime \prime}(B) / e^{i \theta} f^{\prime}(B)\right]$ nicht von $\theta$ abhängt, dürfen wir annehmen, $\mathrm{da} \mathrm{g}^{\prime}(B)$ reell und positiv ist. Wählen wir noch zusätzlich $B>0$, so erhalten wir die Differentialgleichung

$$
w^{\prime}(t)^{2} \frac{1+w(t)^{2}}{w(t)^{3}}<0
$$

Die negative reelle Achse und die rechte Hälfte des Einheitskreises sind Lösungskurven von (7). Die übrigen Lösungskurven entweder winden sich um den Nullpunkt auf solche Weise, daß sie tangentiell zur negativen reellen Achse im Nullpunkt beginnen und enden, oder sie kommen vom Äußeren des Einheitskreises, schneiden die linke Hälfte des Einheitskreises, und enden ebenfalls im Nullpunkt. Wir vermuten, daß die Kurve $\Gamma_{g}$ eine Strecke auf der negativen reellen Achse ist.

Es sei nun $g$ eine Extremalfunktion für das Minimalproblem:

$$
\mathfrak{R}\left(1+B \frac{g^{\prime \prime}(B)}{g^{\prime}(B)}\right)=\min _{f \in F_{B}, b} \Re\left(1+B \frac{f^{\prime \prime}(B)}{f^{\prime}(B)}\right)
$$


In den obigen Ungleichungen brauchen wir nur alle Ungleichheitszeichen umzukehren. Insbesondere erhalten wir für den Spezialfall $B>0, b=0, g^{\prime}(B)>0$, daß die Kurve $\Gamma_{g}$ der Differentialgleichung

$$
w^{\prime}(t)^{2} \frac{1+w(t)^{2}}{w(t)^{3}}>0
$$

genügt. Spiegelt man die Lösungskurven von (7) in der imaginären Achse, so erhält man die Lösungskurven von (8). Wir vermuten, daß $\Gamma_{g}$ ein Bogen (symmetrisch in bezug auf die negative reelle Achse) einer Lösungskurve ist, die sich um den Nullpunkt windet.

\section{Krümmung}

Eine Funktion $f \in F_{B, b}$ bildet den Kreis $|z|=|B|$ auf eine Kurve $C$ ab, die den Punkt $w=b$ enthält. Die Krümmung von $C$ im Punkt $w=b$ ist durch den Ausdruck

$$
K_{B, b, f}=\frac{1}{\left|B f^{\prime}(B)\right|} \mathfrak{R}\left(1+B \frac{f^{\prime \prime}(B)}{f^{\prime}(B)}\right)
$$

gegeben. Wir fragen nach den Extremwerten der Größe $K_{B, b, f}$ für Funktionen der Klasse $F_{B, b}$.

Da die Klasse $F_{B, b}$ kompakt ist, existiert ein $g \in F_{B, b}$ mit der Eigenschaft, daß

$$
K_{B, b, g}=\max _{f \in F_{B, b}} K_{B, b, f}
$$

Ein Vergleich von $g$ mit den Funktionen $U_{\rho} \circ g$ führt zu der Ungleichung

$$
\begin{aligned}
\Re\left\{a \rho ^ { 2 } \left[c\left(\frac{1-\bar{b} w_{0}}{b-w_{0}}\right)^{2}\right.\right. & +\frac{2 B g^{\prime}(B)\left(1-\bar{b} w_{0}\right)^{2}\left(1+|b|^{2}-2 \bar{b} w_{0}\right)}{\left(1-|b|^{2}\right)\left(b-w_{0}\right)^{3}} \\
& \left.\left.+\frac{2 \overline{B g^{\prime}(B)}\left(1-\bar{b} w_{0}\right)}{\left(1-|b|^{2}\right)\left(b-w_{0}\right)}\right]+O\left(\rho^{3}\right)\right\} \leqq 0,
\end{aligned}
$$

wobei $c=\mathfrak{R}\left[1+B g^{\prime \prime}(B) / g^{\prime}(B)\right]$. Ohne Beschränkung der Allgemeinheit dürfen wir annehmen, daß $g^{\prime}(B)>0$. Setzen wir $b=0$, so vereinfacht sich die obige Ungleichung zu dem Ausdruck

$$
\Re\left\{a \rho^{2}\left[\frac{c}{w_{0}^{2}}-\frac{2 B g^{\prime}(B)}{w_{0}^{3}}-\frac{2 \bar{B} g^{\prime}(B)}{w_{0}}\right]+O\left(\rho^{3}\right)\right\} \leqq 0 .
$$

Folglich genügt die Kurve $\Gamma_{g}$ der Differentialgleichung $w^{\prime}(t)^{2} s(w(t))>0$, wobei

$$
s(w)=-w^{-3}\left(2 B g^{\prime}(B)-c w+2 \bar{B} g^{\prime}(B) w^{2}\right) \text {. }
$$

Mit der Normalisierung $B>0$ erhalten wir die Differentialgleichung

$$
\frac{w^{t}(t)^{2}}{w(t)^{3}}\left(1-A w(t)+w(t)^{2}\right)<0
$$

wobei $A=c\left[2 B g^{\prime}(B)\right]^{-1}>0$. Die negative reelle Achse ist offensichtlich eine Lösungskurve, und die übrigen Lösungskurven ähneln denen von Differential- 
gleichung (7). Wir vermuten, daß mit den obigen Normalisierungen die Kurve $\Gamma_{g}$ ein Stück der negativen reellen Achse ist.

Es sei nun $g$ eine Funktion mit der Eigenschaft, daß $K_{B, b, g}=\min _{f \in F_{B, b}} K_{B, b, f}$. Wir dürfen wieder annehmen, daß $g^{\prime}(B)$ positiv ist, und wir brauchen in allen Ungleichungen nur die Ungleichheitszeichen umzukehren. Aber in diesem Fall ist $c$ (und somit $A$ ) nicht notwendig positiv. Für die Normalisierungen $B>0$ und $b=0$ erhalten wir die Differentialgleichung

$$
\frac{w^{\prime}(t)^{2}}{w(t)^{3}}\left(1-A w(t)+w(t)^{2}\right)>0 .
$$

Die Lösungskurven von (9) sind verschieden für $A \geqq-2$ und für $A<-2$. Aber wegen der Beziehung $A=K_{B, 0, g} / 2$ ist die Kurve $\Gamma_{g}$ in jedem Fall ein Bogen, symmetrisch in bezug auf die negative reelle Achse, der sich zur rechten Halbebene hin biegt.

\section{Literatur}

1. Duren, P.L.: Distortion in certain conformal mappings of an annulus. Michigan Math. J. 10, 431-441 (1963).

2. - Schiffer, M.: A variational method for functions schlicht in an annulus. Arch. Rat. Mech. Analysis 9, 260-272 (1962).

3. Gaier, D.: Untersuchungen zur Durchführung der konformen Abbildung mehrfach zusammenhängender Gebiete. Arch. Rat. Mech. Analysis 3, 149-178 (1959).

4. - Huckemann, F.: Extremal problems for functions schlicht in an annulus. Arch. Rat. Mech. Analysis 9, 415-421 (1962).

5. Gehring, F. W., af Hällström, G.: A distortion theorem for functions univalent in an annulus. Ann. Acad. Sci. Fennicae, Ser. A. I. (Math.) 325, 14 pp. (1963).

6. Huckemann, F.: Úber einige Extremalprobleme bei konformer Abbildung eines Kreisringes. Math. Z. 80, 200-208 (1962).

7. - On Schiffer's variational lemma. Arch. Rat. Mech. Analysis 22, 310-312 (1966).

8. - Extremal elements in certain classes of conformal mappings of an annulus. Acta Math. 118, 193-221 (1967).

9. Jenkins, J.A.: Univalent functions and conformal mapping. Second printing corrected. Ergebnisse der Mathematik und ihrer Grenzgebiete, N.F. 18. Berlin-Heidelberg-New York: Springer 1965.

10. Koppenfels, W.von, Stallmann, F.: Praxis der konformen Abbildung. 1. Aufl. Die Grundlehren der mathematischen Wissenschaften in Einzeldarstellungen, Bd. 100. Berlin-GöttingenHeidelberg: Springer 1959.

11. McLaughlin, R.: Extremal problems for functions univalent in an annulus. Dissertation, The University of Michigan, Ann Arbor, Michigan, 1968.

12. Nehari, Z.: Conformal mapping, 1. Aufl. New York: McGraw-Hill 1952.

13. Schiffer, M.: A method of variation within the family of simple functions. Proc. London Math. Soc. (2) 44, 432-449 (1938).

Prof. R. McLaughlin

Department of Mathematics

University of Michigan, Flint College

Flint, Michigan 48503, USA 